\title{
To Cooperate or Not: A Capacity Perspective
}

\author{
Li Wang, Lingkun Kong, Soon Xin Ng and Lajos Hanzo
}

\begin{abstract}
It is widely recognized that differential decode-andforward (DDF) cooperative transmission scheme is capable of providing a superior performance compared to classic direct transmissions employing differential detection, where no channel coding is used. However, the diversity gains achieved by the cooperative system become modest in practical channel coded scenarios, where the interleaving and channel coding gains dominate. Therefore, when a cooperative wireless communication system is designed to approach the maximum achievable spectral efficiency by taking the cooperation-induced multiplexing loss into account, it is not obvious, whether or not the relay-aided system becomes superior to its direct-transmission based counterpart, especially, when advanced channel coding techniques are employed. Hence in this paper the capacity of the single-relayassisted DDF based cooperative system was studied in comparison to that of its direct-transmission based counterpart in order to answer the above-mentioned dilemma.
\end{abstract}

\section{BACKGROUND AND MOTIVATION}

The technological advances in integrated circuits and radiofrequency electronics facilitate the employment of ever more sophisticated signal processing and coding algorithms. At the same time, it is increasingly important to find energy- and bandwidth-efficient solutions for reliable digital communication over time-varying wireless channels. Multiple antenna aided diversity techniques [1] constitute powerful arrangements of mitigating the deleterious effects of fading, hence improving the end-to-end system performance, which is usually achieved by multiple co-located antenna elements at the transmitter and/or receiver. However, it is often impractical for the mobile to employ a large number of antennas for the sake of achieving a diversity gain due to its limited size. Fortunately, in multi-user wireless systems cooperating mobiles may share their antennas in order to achieve uplink transmit diversity by formig a virtual antenna array (VAA) in a distributed fashion $[2,3]$. On the other hand, in order to avoid channel estimation for a VAA-aided system, which may impose both an excessive complexity and a high pilot overhead, especially in mobile environments associated with relatively rapidly fluctuating channel conditions, differentially encoded transmissions combined with non-coherent detection and hence requiring no channel state information (CSI) at the receiver becomes an attractive design alternative, leading to differential modulation assisted cooperative communications [4-6].

In point-to-point communication systems using a singleantenna or co-located multiple antennas, it is feasible to achieve a high spectral efficiency by approaching Shannon's capacity limit with the aid of channel coding $[7,8]$. However, in contrast to the well-understood limitations of point-to-point single-user transmissions, researchers are only beginning to understand the fundamental performance limits of wireless multiuser networks, such as, for example the differentially

The authors are with the school of ECS, University of Southampton, Southampton, SO17 1BJ, UK (e-mail: \{1w05r,lk06r,sxn,lh $\} @$ ecs.soton.ac.uk). Acknowledgements: The finacial support of the EPSRC UK under the auspices of the UK-India centre of Excellence in Wireless Communications and that of the EU Optimix project is gratefully acknowledged. modulated cooperative cellular uplink. Naturally, in the channel uncoded context, the resultant cooperative system performance is expected to be better than that of the non-cooperative transmission $[5,6]$, owing to the cooperative diversity gains as well as the path loss reduction.

However, the cooperative diversity gains promised by the cooperative system considered are actually achieved at the cost of suffering a significant so-called multiplexing loss compared to direct transmissions, imposed by the half-duplex communications of practical transceivers. Furthermore, the cooperative diversity gains achieved by the relay-aided system over its direct-transmission based counterpart may become modest in practical channel coded scenarios, where the interleaving and channel coding gains dominate. Therefore, in the interest of achieving a high spectral efficiency, we have to answer the grave fundamental question, whether it is worth introducing cooperative mechanisms into the development of wireless networks, such as the cellular voice and data networks.

The outline of the paper is as follows. Sections II and III specify the system's architecture and the channel model. In order to answer the above-mentioned question about the ultimate spectral efficiency of repetition-based cooperative relay-aided systems, the fundamental performance limits of the non-coherent detection aided direct-transmission based system will be first studied in Section IV. Then, in Section V the non-coherent Disrecte-input Continuous-output Memoryless Channel's (DCMC) capacity $[9,10]$ of the differential decodeand-forward (DDF) assisted cooperative system [6] will be investigated in comparison to that of its direct-transmission based counterpart.

\section{System ARChitecture}

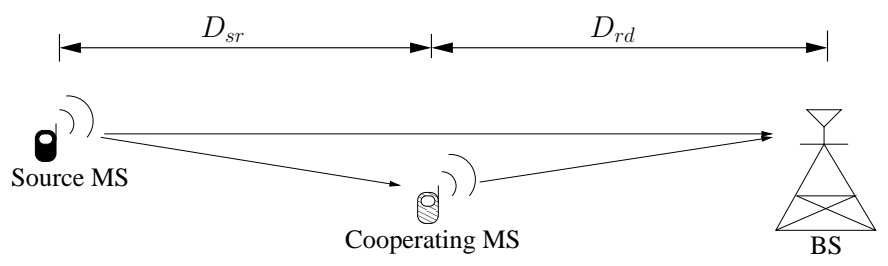

Fig. 1. Single-relay-aided cooperative cellular uplink.

The differentially modulated TDMA cellular uplink is considered without any loss of generality, where no CSI estimation is required. For the sake of simplicity, we consider a singlerelay-assisted scenario of Figure 1, where only one coopearting mobile station (MS) is activated in order to decode and reencode the signal received from the source MS prior to forwarding the signal to the base station (BS). The employment of a single antenna for each terminal is assumed, owing to the cost- and size-constraints of portable transceivers. Since the emphasis in this treatise is on investigating the achievable network capacity of a general repetition-coded cooperative scenario, we simply assume here that the total transmit power is equally divided between the source MS and the single cooperating RS, which is assumed to be located half-way between the source MS and the BS, as depicted in Figure 1. 
More specifically, for the sake of analytical tractability, we simply assume that the sum of the normalized distances $D_{s r}$ between the source MS and the RS, and that between the $\mathrm{RS}$ and the BS, which is represented by $D_{r d}$, is equal to the normalized distance $D_{s d}$ between the source MS and the BS. Naturally, the normalized SD-distance is equal to unity. As a result, observe in Figure 1 that we have: $D_{s r}=D_{r d}=$ $\frac{1}{2} D_{s d}=1$. Furthermore, the normalized average power $\sigma_{i, j}^{2}$ at the output of the channel is inversely proportional to the inter-node distance $D_{i, j}$, which may be rewritten as follows:

$$
\sigma_{i, j}^{2}=D_{i, j}^{-v}, \quad i, j \in\{s, r, d\},
$$

where $v$ denotes the path-loss exponent [11] and the subscripts $s, r$ and $d$ represent the source, relay and destination, respectively.

\section{Channel Model}

Throughout this paper we assume that the complex-valued basedband signals undergo Rayleigh fading, which is modelled by multiplying the transmitted signal by a complexvalued Gaussian random variable. In order to provide a good approximation for TDMA-based cooperative systems and to facilitate the study of the non-coherent detection-based channel capacity, we consider a block-fading Rayleigh channel, where the fading coefficients are assumed to change in an independent and identically-distributed (i.i.d.) manner from block to block. On the other hand, instead of employing the standard block-fading channel, where the fading coefficient remains constant over the duration of several consecutive symbol periods, we consider here a time-selective block-fading model [12], which includes the former as a special case. In the time-selective block-fading channel model considered, the channel's envelope exhibits correlation within a transmission block according to the Doppler frequency induced by the relative movement of the tranceivers.

Consider a single-antenna-assisted point-to-point transmission scheme communicating over a block-fading channel, which exhibits a correlated envelope for the duration of $T_{b}$ consecutive symbols. Then, the received signal may be formulated as:

$\begin{aligned} \mathbf{y} & =\mathbf{S}_{d} \mathbf{h}+\mathbf{w}, \\ \text { where we have } \mathbf{y} & =\left[\begin{array}{ll}y_{1}, & y_{2}, \cdots, y_{T_{b}}\end{array}\right]_{T}^{T}, \mathbf{h}=\end{aligned}$ $\left[h_{1}, h_{2}, \cdots, h_{T_{b}}\right]^{T}$, and $\mathbf{w}=\left[w_{1}, w_{2}, \cdots, w_{T_{b}}\right]^{T}$ representing the received signal column vector, the fading coefficient column vector obeying a complex-valued Gaussian distribution $\mathcal{C N}\left(0, \sigma_{h}^{2}\right)$ and the Gaussian noise column vector having a distribution of $\mathcal{C N}\left(0,2 \sigma_{w}^{2}\right)$, respectively. The diagonal matrix $\mathbf{S}_{d}$ in Eq.(2) hosts the $T_{b}$ consecutively transmitted signals within a block, which may be expressed as $\mathbf{S}_{d}=\operatorname{diag}\{\mathbf{s}\}$, where we have $\mathbf{s}=\left[s_{1}, s_{2}, \cdots, s_{T_{b}}\right]^{T}$. Furthermore, in the cooperative communication scenario of Figure 1, the normalized channel fading variance $\sigma_{h}^{2}$ of each link was formulated in Eq.(1) by taking the path-loss into account. Given the assumption of Rayleigh fading, $\mathbf{h}$ is a zero-mean complex-valued Gaussian vector with a $\left(T_{b} \times T_{b}\right)$-element covariance matrix $\Sigma_{h}$, which may be written as:

$$
\begin{aligned}
\Sigma_{h} & =\mathcal{E}\left\{\mathbf{h h}^{H}\right\} \\
& =\sigma_{h}^{2} \cdot\left[\begin{array}{cccc}
\varphi^{t}[0] & \varphi^{t}[1] & \cdots & \varphi^{t}\left[T_{b}-1\right] \\
\varphi^{t}[-1] & \varphi^{t}[0] & \cdots & \varphi^{t}\left[T_{b}-2\right] \\
\vdots & \vdots & \ddots & \vdots \\
\varphi^{t}\left[1-T_{b}\right] & \varphi^{t}\left[2-T_{b}\right] & \cdots & \varphi^{t}[0]
\end{array}\right],
\end{aligned}
$$

where $\varphi^{t}[\kappa]$ represents the channel's autocorrelation function, which can be expressed as: $\varphi^{t}[\kappa] \triangleq \mathcal{E}\left\{h[n+\kappa] h^{*}[n]\right\}=$ $J_{0}\left(2 \pi f_{d} \kappa\right)$, with $J_{0}(\cdot)$ denoting the zeroth-order Bessel function of the first kind and as usual, $f_{d}$ represents the normalized Doppler frequency.

\section{NON-COHERENT CHANNEL CAPACITY FOR POINT-TO-POINT LINKS}

Let us now first focus our attention on the non-coherent DCMC capacity of the classic single-antenna-assisted pointto-point communication scenario, based on which the noncoherent DCMC network capacity of the DDF-aided cooperative system will be studied in Section V. The PDF of the received signal vector $\mathbf{y}$ in Eq. (2) was conditioned on the transmitted signal vector $\mathbf{s}$, which may be readily expressed as [13]:

$$
p(\mathbf{y} \mid \mathbf{s})=\frac{\exp \left(-\mathbf{y}^{H} \Psi^{-1} \mathbf{y}\right)}{\operatorname{det}(\pi \Psi)}
$$

where we have $\Psi=\mathcal{E}\left\{\mathbf{y} \mathbf{y}^{H} \mid \mathbf{s}\right\}=\mathbf{S}_{d} \Sigma_{h} \mathbf{S}_{d}^{H}+2 \sigma_{w}^{2} \mathbf{I}_{T_{b}}$ with $\mathbf{I}_{T_{b}}$ denoting the $\left(T_{b} \times T_{b}\right)$-element identity matrix.

Since differentially encoded modulation schemes, such as DQPSK, are assumed to be employed at MSs, and each element $s_{i}$ of the transmitted signal vector $\mathbf{s}$ is chosen independently from a finite constellation set $\mathcal{M}_{c}$ with equal probabilities, the non-coherent DCMC capacity can be expressed as a function of the SNR as follows:

$$
C(\mathrm{SNR})=H(\mathbf{y})-H(\mathbf{y} \mid \mathbf{s})
$$

where $H$ represents the differential entropy [14] of a random variable $\mathbf{x}$ defined as $H(\mathbf{x})=-\int p(\mathbf{x}) \log _{2} p(\mathbf{x}) d \mathbf{x}$, with $p(\cdot)$ denoting the corresponding PDF. According to [14], the differential entropy $H(\mathbf{y} \mid \mathbf{s})$ may be readily calculated as:

$$
H(\mathbf{y} \mid \mathbf{s})=\log \operatorname{det}(\pi \mathrm{e} \Psi) \text { bits. }
$$

On the other hand, the entropy $H(\mathbf{y})$ of the continuous-valued faded and noise-contaminated received signal vector $\mathbf{y}$ cannot be evaluated in a closed form. When the fading block size $T_{b}$ over which the fading envelope is assumed to be correlated is limited, a practical approach to the numerical evaluation of $H(\mathbf{y})$ is to carry out Monte-Carlo integration as follows [10]:

$$
\begin{aligned}
H(\mathbf{y}) & =-\int p(\mathbf{y}) \log p(\mathbf{y}) d \mathbf{y} \\
& =-\mathcal{E}\left\{\log _{2}\left(\frac{1}{M_{c}^{T_{b}} \operatorname{det}(\pi \Psi)} \sum_{\tilde{\mathbf{s}} \in \chi} \exp \left(-\mathbf{y}^{H} \Psi^{-1} \mathbf{y}\right)\right)\right\},
\end{aligned}
$$

where $\chi$ is the set of all $M_{c}^{T_{b}}$ hypothetically transmitted

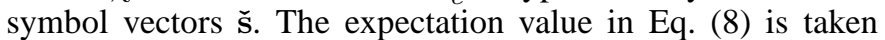
with both respect to different channel impulse response (CIR) realizations and to the noise.

The non-coherent DCMC capacity of the standard blockfading channel computed using the DQPSK modulation scheme is plotted in Figure 2(a) for various fading block sizes of $T_{b}=2,4$ and 7. As observed in Figure 2(a), although an identical differential modulation scheme (DQPK) is employed, the maximum achievable spectral efficiency associated with a sufficiently high SNR is dependent on the fading block size $T_{b}$. This is not unexpected, since the differential signaling process commences by transmitting a reference symbol for each fading block [9], which does not contain any information. This reference symbol constitutes unexploited transmission 

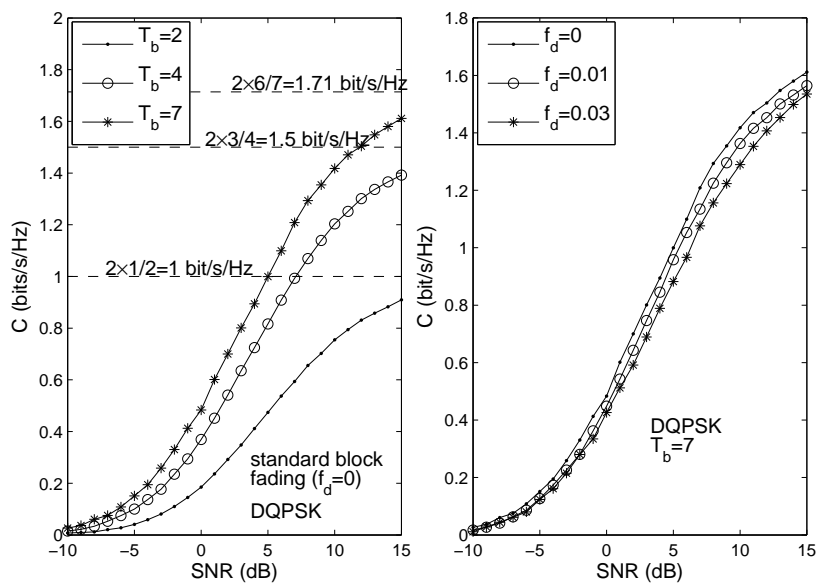

(a) For various fading block size (b) For various Doppler frequencies

Fig. 2. Non-coherent DCMC capacity of the single-input single-output timeselective block-fading channel.

overhead, which hence imposes a diminishing capacity erosion, as $T_{b}$ is increased. Thus, as the SNR increases, the achievable bandwidth efficiency $\eta_{\max }$ saturates at:

$$
\eta_{\max }=\log _{2} M_{c} \times\left(T_{b}-1\right) / T_{b}, \quad \text { bits } / \mathrm{s} / \mathrm{Hz},
$$

which approahes that of the coherent detection aided transmission scheme, if the fading block size $T_{b}$ increases towards infinity.

On the other hand, according to [12], the predictability of the channel is characterized by the rank $Q$ of the channel's covariance matrix $\Sigma_{h}$ formulated in Eq. (3). For example, the block-fading channel, where the fading envelope remains constant over the entire fading block is associated with the most predictable fading envelope, when the channel's covariance matrix has a rank of $Q=1$. By contrast, the fading process has a finite differential entropy and becomes less predictable, when we have $Q=T_{b}$. Figure 2(b) compares the non-coherent DCMC capacity of the time-selective block-fading channel computed from Eq. (5) for the DQPSK modulation scheme and for various normalized Doppler frequencies. When we have an increased channel unpredictability owing to increased Doppler frequency, a capacity loss is observed in Figure 2(b). Hence, the observation of Figure 2(a) and 2(b) suggests that the non-coherent DCMC capacity of a time-seletive blockfading channel is dependent on both the fading block size $T_{b}$ and the fading correlation over blocks characterized by the corresponding covariance matrix $\Sigma_{h}$.

\section{Relay-Aided Cooperative Network CAPACITY}

Let us now commence to investigating the DCMC capacity of the differentially modulated single-relay-aided cooperative system of Figure 1. We first define the two-hop singlerelay-aided network's capacity as the maximum achievable rate attained during the transmission of the source $M S$ in the broadcast phase, namely, Phase I, which consists of $L_{s}$ symbol periods, and an independent transmission by the $R S$ during the relaying phase, namely, Phase II, when $L_{r}$ symbols are transmitted. Initially a perfect SR link is assumed in Section V-A in order to guarantee "error-free" relaying. Thus the above-mentioned network capacity is termed as the cooperative system's DCMC capacity, which is not affected or constrained by the quality of the SR link. Hence we

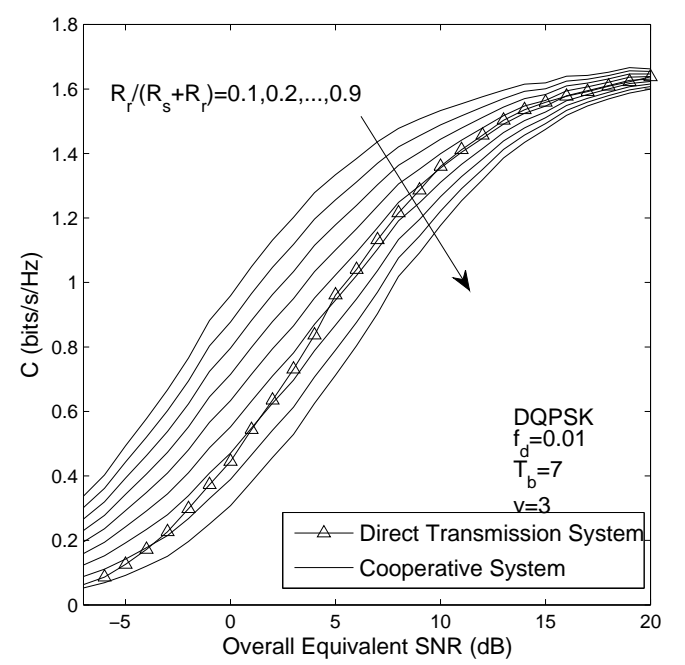

Fig. 3. The single-relay-assisted cooperative system's constant code-rate-ratio based DCMC capacity curves for the 'perfect-SR-link' based scenario.

refer to it in parlance as the 'perfect-SR-link' based capacity. By contrast, in Section V-B its 'imperfect-SR-link' based counterpart will be investigated by considering the specific performance limitations imposed by the potentially error-prone SR link.

\section{A. Perfect-SR-Link Based DCMC Capacity}

According to the above definition, under the assumption of an equal-power-allocation and a mid-point RS location, the corresponding network's 'perfect-SR-link' based DCMC capacity may be formulated as:

$$
\begin{aligned}
& C_{D C M C}^{\text {coop }}\left(\alpha, \mathrm{SNR}_{t}^{\text {overall }}\right) \\
= & \alpha C_{D C M C}^{\text {sd }}\left(\mathrm{SNR}_{e}^{s}\right)+(1-\alpha) C_{D C M C}^{r d}\left(\mathrm{SNR}_{e}^{r}\right), \\
= & \alpha C_{D C M C}\left(\mathrm{SNR}_{e}^{\text {overall }} / 2\right) \\
+ & (1-\alpha) C_{D C M C}\left[\mathrm{SNR}_{e}^{\text {overall }} / 2+10 \log _{10}\left(\sigma_{r d}^{2}\right)\right],
\end{aligned}
$$

where

$$
\alpha \triangleq \frac{L_{s}}{L_{s}+L_{r}}=\frac{R_{r}}{R_{s}+R_{r}},
$$

since the ratio of the differential-encoding frame lengths used by the source and relay is inversely proportional to the ratio of the channel code rate $\left(R_{s}, R_{r}\right)$ employed by the two. In Eq. (11), $\sigma_{r d}^{2}$ characterizes the reduced-path-loss related power-gain, which was given by Eq. (1) and $C_{D C M C}(\cdot)$ represents the SISO non-coherent DCMC capacity formula of Eq. (5). Furthermore, $\mathrm{SNR}_{e}^{s}$ and $\mathrm{SNR}_{e}^{r}$ in Eq. (11) represent the equivalent SNRs at the source and relay transmitters, respectively, which have the following relationship with the network's overall equivalent $\mathrm{SNR}, \mathrm{SNR}_{e}^{\text {overall }}$, as $\mathrm{SNR}_{e}^{s}=$ $\mathrm{SNR}_{e}^{r}=\frac{1}{2} \mathrm{SNR}_{e}^{\text {overall }}$.

Therefore, in contrast to the independence of the DCMC capacity of the channel code rate employed in the scenario of the conventional direct-transmission based system, the DCMC capacity of the relay-aided cooperative system is dependent on the ratio $\frac{R_{s}}{R_{r}}$ of the channel code rates employed by the source and relay or, equivalently, dependent on $\alpha$. In Figure 3 the cooperative system's DCMC capacity curves associated with different values of $\alpha$ are depicted based on Eq. (11) 


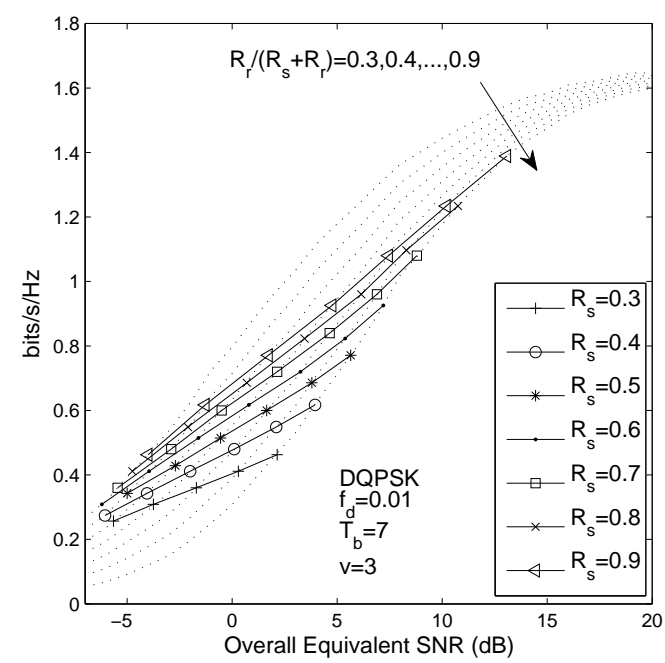

Fig. 4. The single-relay-assisted cooperative system's constant- $R_{s}$ based DCMC capacity curves.

for the 'perfect-SR-link' scenario at a constant code-rateratio in comparison to the DCMC capacity curve of the direct-transmission based system. As observed in Figure 3, the cooperative system's DCMC capacity is gradually decreased as $\alpha$ is increased. This is not unexpected, since the weight of the second term in Eq. (11) decreases as that of the first term increases, while the second term is typically larger than the first term owing to the reduced-path-loss related power gain. Furthermore, since the half-duplex constraint imposes a potentially substantial multiplexing loss, the system's constant coderate-ratio based 'perfect-SR-link' associated DCMC capacity may become even lower than that of the direct-transmission based system, as seen in Figure 3, if both the overall equivalent SNR and $\alpha$ is sufficiently high.

On the other hand, given the channel code rates $\left(R_{s}, R_{r}\right)$ employed by the source and relay, the resultant bandwidth efficiency, $\eta$, may be expressed as:

$$
\eta=\frac{L_{s} R_{s}}{L_{s}+L_{r}} \frac{T_{b}-1}{T_{b}} \log _{2} M_{c}=\alpha R_{s} \frac{T_{b}-1}{T_{b}} \log _{2} M_{c} .
$$

Hence, by fixing the value of $R_{s}$ and varying that of $\alpha$, the resultant bandwidth efficiency $\eta$ can be calculated using Eq. (13). Based on Eq. (13) the corresponding minimum overall equivalent SNR required by near-error-free transmissions may be found with the aid of the constant code-rate-ratio based 'perfect-SR-link' associated DCMC capacity curves of Figure 3.

Consequently, the cooperative system's constant- $R_{s}$ 'perfect-SR-link' based DCMC capacity curves were plotted from Eq. (13) based on Figure 3 for various values of $R_{s}$ in Figure 4, where we observe that the capacity increases as $R_{s}$ increases. However, all the constant- $R_{s}$-related capacity curves depicted in Figure 4 would intersect the capacity curve of the direct-transmission based system plotted in Figure 3, if the overall equivalent SNR becomes sufficiently high. Therefore, based on the observation of Figures 3 and 4, we may state that although the cooperative system's capacity increases steadily as the overall equivalent SNR increases, it might remain lower than that of its direct-transmission based counterpart, even under the assumption of an idealized error-free SR link, if both $R_{s}$ and $\alpha$ are of relatively high
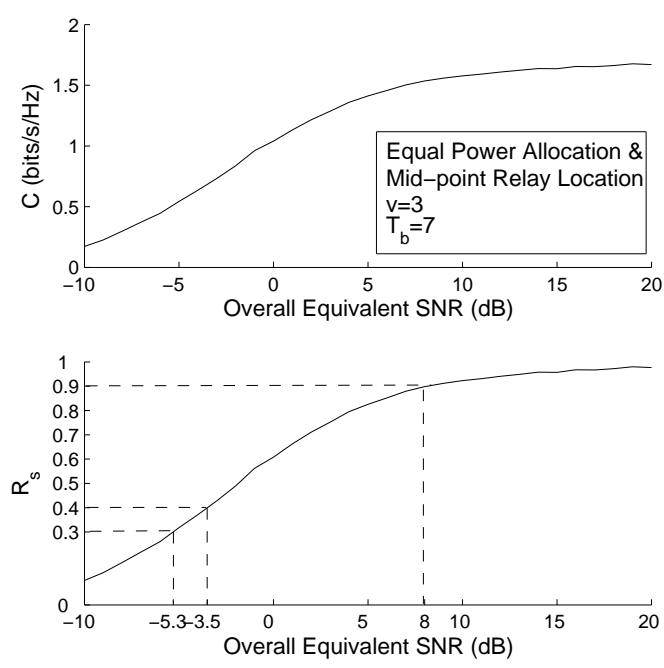

Fig. 5. Non-coherent DCMC capacity of the SR link and its corresponding capacity-achieving channel code rate $R_{s}$ employed by the source MS.

values.

\section{B. ImPerfect-SR-Link Based DCMC Capacity}

Until now the single-relay-assisted DDF cooperative system's capacity has been investigated under the assumption of an idealized error-free SR link. However, in practice the wireless channel connecting the source and relay MSs is typically far from perfect and its quality plays an important role in determining the overall cooperative network's achievable performance, as observed in [6] in the context of uncoded scenarios. Furthermore, in order to create a near-capacity design for the overall cooperative system, near-capacity transmission over the potentially error-infested SR link during the broadcast Phase $I$ is a natural prerequisite, which in turn leads to the investigation of the performance limitations imposed by the SR link on the overall cooperative system.

Under the assumption of equal-power-allocation and a midpoint relay location, the non-coherent DCMC capacity of the SR link may be expressed as:

$C_{D C M C}^{s r}\left(\mathrm{SNR}_{e}^{\text {overall }}\right)=C_{D C M C}\left(\frac{\mathrm{SNR}_{e}^{\text {overall }}}{2}+10 \log _{10}\left(\sigma_{s r}^{2}\right)\right)$,

where again $C_{D C M C}(\cdot)$ was formulated in Eq. (5). Hence, the non-coherent DCMC capacity of the SR link may be plotted versus the overall equivalent SNR, as shown in Figure 5. Then, we can calculate the capacity-achieving channel code rate employed by the source as:

$$
R_{s, \text { capacity }}\left(\mathrm{SNR}_{e}^{\text {overall }}\right)=\frac{T_{b} \cdot C_{D C M C}^{s r}\left(\mathrm{SNR}_{e}^{\text {overall }}\right)}{\left(T_{b}-1\right) \cdot \log _{2} M_{c}},
$$

which is also depicted versus the overall equivalent SNR in Figure 5. Therefore, the minimum overall equivalent SNR corresponding to a certain value of $R_{s}$, which faciliates nearerror-free information delivery from the source to the relay, may be observed in Figure 5. These minimum overall equivalent SNRs characterize the performance limits imposed by the practical imperfect SR link on the entire cooperative system, when the corresponding rate $R_{s}$ is employed by the source. 


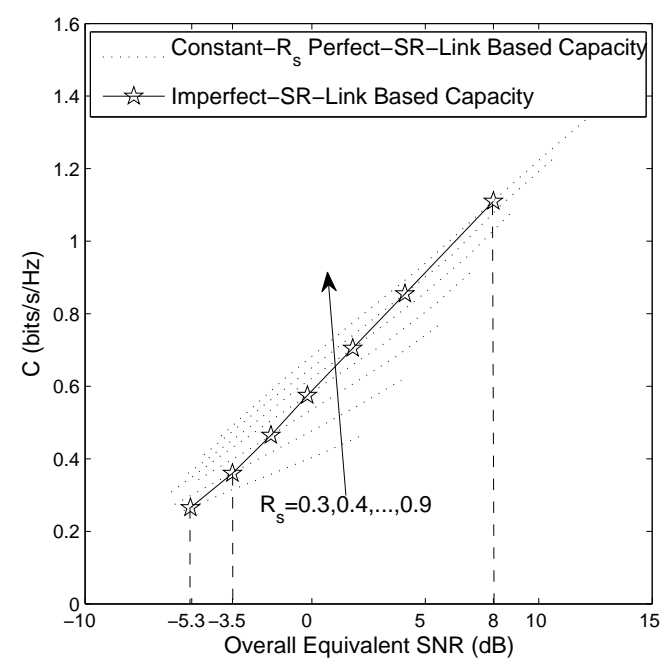

Fig. 6. Imperfect-SR-link based DCMC capacity.

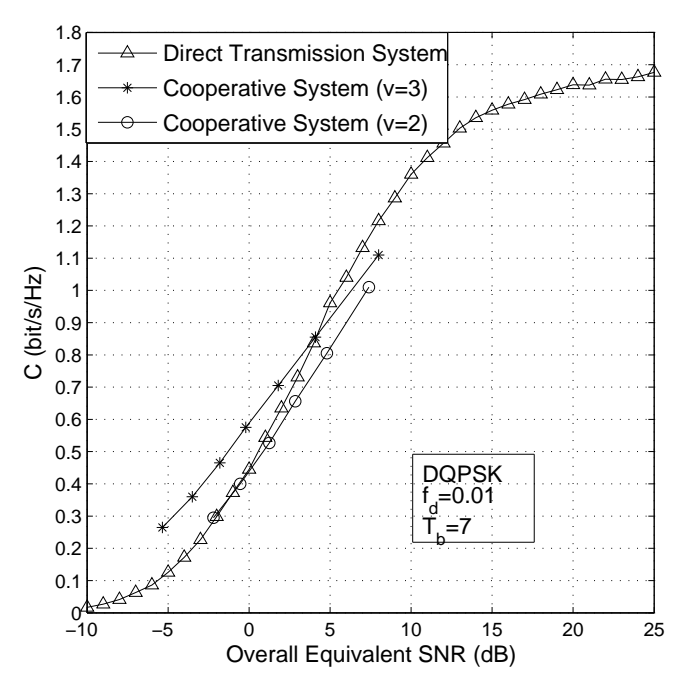

Fig. 7. Capacity comparison of the single-relay-aided cooperative system and its direct-transmission based counterpart.

Given these minimum overall equivalent SNRs associated with different values of $R_{s}$, we can now draw the cooperative system's 'imperfect-SR-link' related DCMC capacity based on the constant- $R_{s}$ 'perfect-SR-link' based DCMC capacity curves of Figure 4. More specifically, observe in Figure 6 that in order to find, for example, the cooperative system's 'imperfect-SR-link' based DCMC capacity for $R_{s}=0.3$, we locate the particular point on the constant- $R_{s}$ 'perfect-SRlink' based DCMC capacity curve associated with $R_{s}=0.3$, whose horizontal coordinate is equal to the corresponding minimum overall equivalent SNR of $-5.3 \mathrm{~dB}$ found previously in the context of Figure 5. Then, the vertical coordinate of the point is the 'imperfect-SR-link' based DCMC capacity of the cooperative system for $R_{s}=0.3$ or when we have $\mathrm{SNR}_{e}^{\text {overall }}=-5.3 \mathrm{~dB}$.

\section{CAPACITY COMPARISON AND DISCUSSIONS}

In order to gain an insight into the benefits of the single-relay-assisted DDF cooperative system over its conventional point-to-point direct-transmission based counterpart from a pure capacity perspective, the 'imperfect-SR-link' based DCMC capacity of the cooperative system associated with both $v=2$ and $v=3$ is depicted in comparison to that of the direct-transmission based one in Figure 7. It may be observed in the figure that when the overall equivalent SNR is relatively low, the single-relay-assisted cooperative system exhibits a significantly higher capacity than its directtransmission based counterpart in typical urban cellular radio scenarios, e.g. when having a path-loss exponent of $v=3$. However, the achievable capacity gain may be substantially reduced if we encounter a free-space propagation scenario [11], i.e. $v=2$, since the reduced-path-loss-related power-gain achieved is insufficiently high to compensate for the significant multiplexing loss inherent in the single-relay-aided half-duplex cooperative system. Moreover, as the overall equivalent SNR increases to a relatively high value, there is no benefit in invoking a single-relay-aided cooperative system, since its capacity becomes lower than that of the conventional pointto-point system.

\section{CONCLUSION}

The non-coherent DCMC capacity of the single-relayassisted DDF cooperative system was investigated. We found that classic direct transmissions might outperform single-relayaided cooperative systems, unless countermeasures, such as successive relaying [15] are invoked, which will be considered in our future work.

\section{REFERENCES}

[1] G. J. Foschini and M. J. Gans, "On limits of wireless communications in a fading environment when using multiple antennas," Wireless Personal Communications, vol. 6, pp. 311-335, Mar. 1998.

[2] J. N. Laneman, D. N. C. Tse, and G. W. Wornell, "Cooperative diversity in wireless networks: Efficient protocols and outage behavior," IEEE Transaction on Information Theory, vol. 50, pp. 3062-3080, Dec. 2004.

[3] K. G. Seddik, A. K. Sadek, W. Su, and K. J. R. Liu, "Outage analysis and optimal power allocation for multinode relay networks," IEEE Signal Processing Letters, vol. 14, pp. 377-380, June 2007.

[4] T. Himsoon, W. Su, and K. J. R. Liu, "Differential transmission for amplify-and-forward cooperative communications," IEEE Signal Processing Letters, vol. 12, pp. 597-600, Sept. 2005.

[5] L. Wang and L. Hanzo, "The amplify-and-forward cooperative uplink using multiple-symbol differential sphere-detection," IEEE Signal Processing Letters, vol. 16, pp. 913-916, Oct. 2009.

[6] L. Wang and L. Hanzo, "The resource-optimized differentially modulated hybrid $\mathrm{AF} / \mathrm{DF}$ cooperative cellular uplink using multiple-symbol differential sphere detection," IEEE Signal Processing Letters, vol. 16, pp. 965-968, Nov. 2009.

[7] M. Tüchler, "Design of serially concatenated systems depending on the block length," IEEE Transactions on Communications, vol. 52, pp. 209218, Feb. 2004

[8] L. Wang, L. Xu, S. Chen, and L. Hanzo, "Three-stage irregular convolutional coded iterative center-shifting $k$-best sphere detection for softdecision SDMAOFDM," IEEE Transactions on Vehicular Technology, vol. 58, pp. 2103-2109, May 2009.

[9] J. G. Proakis, Digital Communications. 4th edition, New York, NY: Mc-Graw-Hill, 2000.

[10] R.-R. Chen, R. Koetter, U. Madhow, and D. Agrawal, "Joint noncoherent demodulation and decoding for the block fading channel: a practical framework for approaching shannon capacity," IEEE Transactions on Communications, vol. 51, pp. 1676-1689, Oct. 2003.

[11] T. S. Rappaport, Wireless Communications Principles and Practise. Pearson Education Asia Limited and Publishing House of Electronics Industry, second ed., 2002.

[12] Y. Liang and V. V. Veeravalli, "Capacity of noncoherent time-selective rayleigh-fading channels," IEEE Transactions on Information Theory, vol. 50, pp. 3095-3110, Dec. 2004.

[13] R.-R. Chen, R. Koetter, and U. Madhow, "Joint noncoherent demodulation and decoding for fast rayleigh fading channels," Conference on Information Sciences and Systems, Mar. 2003.

[14] T. M. Cover and J. A. Thomas, Elements of Information Theory. Second Edition, John Wiley \& Sons, Inc., 2006.

[15] Y. Fan, C. Wang, J. Thompson, and H. V. Poor, "Recovering multiplexing loss through successive relaying using repetition coding," IEEE Transactions on Wireless Communications, vol. 6, pp. 4484-4493, Dec. 2007. 